\title{
Simulation of nanoparticle aggregation process in heterogeneous dispersed systems
}

\author{
Valentyn Olishevskyi, Serhii Vasylenko, \\ Evhen Babko, Sviatoslav Lementar
}

\section{National University of Food Technologies, Kyiv, Ukraine}

Keywords:

Coagulation

Aggregation

Nanoparticles

Diffusion

Dissipation

Article history:

Received 01.12.2019

Received in revised

form 16.03.2020

Accepted 30.06.2020

\section{Corresponding} author:

Valentyn Olishevskyi

E-mail:

valinter@ukr.net

DOI: $10.24263 / 2310-$

1008-2020-8-1-10

\section{Abstract}

Introduction. The study of particle aggregation is relevant and studied in chemical technology, biophysics, in solving problems of purification from aerosol or colloidal contaminants.

Materials and methods. The influence of nanoparticles on the aggregation kinetics of dispersed phases in suspension is considered. Models based on modifications of the particle dynamics method taking into account Van der Waals forces, gravity, Brownie and Stokes forces based on semi-empirical dependences for the rate of aggregation and disaggregation in collisions are used.

Results and discussion. Based on the analysis of the Smolukhovsky equation, molecular kinetic and localisotropic turbulence of Kolmogorov's theories, the dynamics of simultaneous turbulent and Brownian transfer to the process of "rapid coagulation" and the mechanism of influence of nanoparticles on their accumulative ability in colloidal heterogeneous dispersion are proposed.

The developed equations for the rate of kinetic energy scattering in a suspension makes it possible to determine the particle dispersion in which the Brownian diffusion of particles in the coagulation mechanism predominates. This means, the more intense the process of mixing the suspension, the greater the role in the process of coagulation, precipitation and filtration play particles of the nanoscale range, which are included in the rapid movement of the vortex. These results are useful for practical application in control of intensity of coagulation processes in mixing devices.

Conclusions. The mechanism of influence of nanoparticles on their aggregation ability in colloidal heterogeneous disperse systems is offered. 


\section{Introduction}

There is high interest in the study of nanosized particles, due to the fact that they have special mechanical, optical, electrical and magnetic properties that differ from the properties of conventional macroparticles [1-4]. Studies conducted in recent years have shown the high efficiency of the use of colloidal solutions of nanosized particles of biologically active metals in medicine, biology, veterinary medicine and agriculture [3, 6].

The most commonly used are colloidal suspensions of nanoparticles $\mathrm{Al}_{2} \mathrm{O}_{3}, \mathrm{CuO}, \mathrm{SiO}_{2}$, $\mathrm{ZnO}$ with a particle diameter $\mathrm{d}_{\mathrm{p}}=20-60 \mathrm{~nm}$ based on water, glycerin, ethylene glycol and fullerene suspensions $\left(d_{p}=1,6-1,8 \mathrm{~nm}\right)$, nanotubes $\left(d_{p}=5-100 \mathrm{~nm}\right)$, nanocrystals and other particles [7, 8]. Nanofluids have unique thermomechanical, electrical and magnetic properties even at low $(<5 . .10 \%)$ nanoparticle concentrations. Adding $\mathrm{Ch}=1.8 \%$ of nanoparticles $\mathrm{Al}_{2} \mathrm{O}_{3}$ in the coolant leads to a heat increase dissipation from the $\mathrm{PC}$ processor by $32 \%$ [9]. The optimum concentration at which the thermal conductivity of a liquid becomes high and the dynamic viscosity becomes low depends on the nature of the particles, their size, shape, roughness, and surface adhesion. [8, 10]. In the review [11] the dependences of the efficiency of different nanofluids in the range $\mathrm{Re}=1 \ldots 1200$ are given.

It is known that during the operation of such liquids, nanoparticles become unstable and prone to aggregation [12, 13]. To prevent aggregation and subsequent gravitational deposition, nanoparticles are stabilized by methods and substances that are safe to use and provide their chemical and aggregative stability during synthesis [14].

Combining the particles of the dispersed phase into one, when they converge and collide, is the basis of aggregation (coagulation, coalescence). Particle collisions and their adhesion or fusion (in the case of liquid particles and gas bubbles) can be caused by various consequences: chaotic (Brownian or violent) oscillations, convergence under the force of electric, magnetic, gravitational, hydrodynamic and other forces. Random collisions do not always lead to the interaction of particles, because in the dispersion phase they acquire the same surface electric charges and repel each other. Aggregation and coagulation underlie the processes of aggregation-sediment instability of dispersed systems, their stratification into solid and liquid phases, precipitation or transition from sol to gel. The study of particle aggregation is relevant and is studied in chemical technology, biophysics, in solving problems of purification from aerosol or colloidal contaminants. [15-18].

\section{Materials and methods}

This work considers mathematical models of aggregation kinetics in multiphase dispersed systems, investigates the dynamics of simultaneous turbulent and Brownian transfer to the process of "fast coagulation" and the mechanism of influence of nanoparticles on their aggregation ability in colloidal heterogeneous dispersed systems is proposed.

The influence of nanoparticles on the aggregation kinetics of dispersed phases in suspension is considered (Figure 1).

In this work, we study the dynamics of the simultaneous turbulent and Brownian transition to the process of "rapid coagulation" and propose a mechanism for the influence of nanoparticles on the aggregation capacity of suspensions.. 


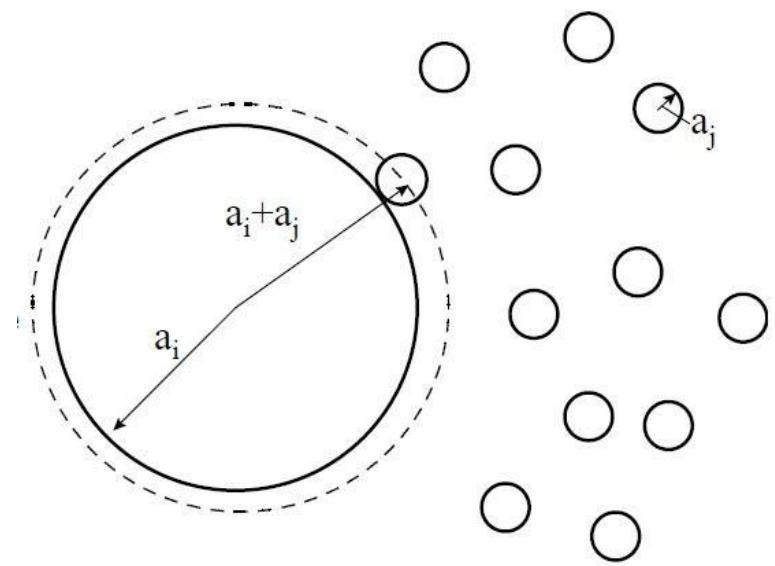

Figure 1. Schematic representation of Brownian coagulation particles:

$a$ - radius of the particle;

$i$ and $j$ - respectively particle and particle flow;

$a_{i}+a_{j}-$ the distance between the centers of the two contacting particles.

The influence of nanoparticles on the aggregation kinetics of dispersed phases in suspension is considered.

Modeling is based on modifications of the particle dynamics method taking into account Van der Waals forces, gravity, Brownie and Stokes forces based on semi-empirical dependences for the rate of aggregation and disaggregation in collisions are used.

\section{Results and discussions}

The concentration of particles is considered to be quite high $(\mathrm{C}<0.05)$, so they do not settle [5]. With additional surface stabilization and the absence of electrostatic interactions, such suspensions can be stable and stay in a suspended state for a long time [3, 4]. When used in microfluidic systems, the number of collisions increases and, due to the high adhesion of particle surfaces, they often form aggregates. Thus, the threshold concentration of $\mathrm{C}$, at which aggregation and sedimentation of particles is observed, is determined by a significant number of factors, but, according to various estimates, close to $\mathrm{C} \sim 0.05$ [3-5].

Thus, in an aqueous suspension of zero-valent metal nanoparticles with $\mathrm{d}_{\mathrm{p}}=20 \mathrm{~nm}$ at a concentration of $2 \mathrm{mg} / 1$ and $60 \mathrm{mg} / \mathrm{l}$ after $10 \mathrm{~min}$, aggregates with an average radius of 125 $\mathrm{nm}$ and $1200 \mathrm{~nm}$, respectively, are formed, which rapidly settle in water [19]. For multiphase dispersed systems (usually underground drivers, drinking water), for aggregation and production of aggregate nanoparticles with $\mathrm{pH}$, ionic strength and available impurities [20]. Zinc oxide nanoparticles of $\mathrm{ZnO}$ form aggregates and precipitate rapidly when $\mathrm{d}_{\mathrm{m}}>50 \mathrm{~nm}$ [21].

The nanoparticle suspension models available in the literature are based on modifications of the particle dynamics method taking into account Van der Waals forces, gravity, Brown and Stokes forces based on semi-empirical dependences for the aggregation rate and disaggregation in collisions. [22-24].

In practical conditions, the most important transport mechanisms of particles are Brownian diffusion caused by their random motion induced by the internal energy of the 
system in the absence of fluid motion. Particle aggregation due to these collisions is known as perikinetic flocculation. Transport of particles due to Brownian motion is important mainly for submicron particles, as other transport mechanisms begin to predominate in the case of large particles.

M. Smolukhovsky [25], considering Brown's coagulation in colloids, estimated the frequency of particle collisions for perikinetic aggregation. Diffusion coefficients for a spherical particle of radius ai are obtained on the basis of the Stokes-Einstein equation [26]:

$$
D_{i}=\frac{k_{B} T}{6 \pi a_{i} \eta^{\prime}}
$$

where $k_{B}$ - Boltzmann's constant, which is defined by Stokes' law $(\gamma=6 \pi \eta a) ; T-$ absolute temperature; $a_{i}$ - particle radius; $\eta$-dynamic viscosity of the dispersion medium.

The total number of collisions per unit volume and per unit time for scattered particles is:

$$
J_{i j}=4 \pi R_{j}\left(D_{i}+D_{j}\right) n_{i} n_{j}
$$

where $R_{i j}$ - the distance between the centers of the particles $i$ and $j$, on which they are supposed to be in contact (fig.1). Assuming that $R_{i j}=a_{i}+a_{j}$, then the total number of collisions will be:

$$
J_{i j}=\frac{2 k_{B} T}{3 \eta} \frac{\left(a_{i}+a_{j}\right)^{2}}{a_{i} a_{j}} n_{i} n_{j}
$$

Velocity constant for collisions between particles $i$ and $j$ with perikinetic aggregation will be [22]:

$$
k_{i j}=\left(\frac{2 k_{B} T}{3 \eta}\right) \frac{\left(a_{i}+a_{j}\right)^{2}}{a_{i} a_{j}} .
$$

Thus, the frequency of particle collisions during Brownian motion increases with the temperature of the dispersion medium, while the effect of particle size is less obvious. When the radii of the particles increase, the diffusion coefficient (1) decreases, and the contact radius, $R_{i j}$ increases in such a way that the effect of particle size is somewhat canceled, and $\frac{\left(a_{i}+a_{j}\right)^{2}}{a_{i} a_{j}} \approx$ has a constant value for different particles.

The movement of a liquid, regardless of whether it is associated with mixing or organized movement of the liquid, can significantly increase the speed of collision between particles, and hence the speed of aggregation. Aggregation through superimposed gradients of mixing rate or fluid flow is known as orthokinetic flocculation.

For the laminar velocity field, M. Smoluchowski's approach predicted that orthokinetic aggregation estimates that particles move by fluid flows with a velocity gradient $G$, and collide as they approach the distance $a_{i}+a_{j}$ between their centers (fig. 1 ).

Therefore, the rate constant for orthokinetic aggregation is estimated as:

$$
k_{i, j}=\left(\frac{4}{3}\right) G\left(a_{i}+a_{j}\right)^{3} .
$$

This method of aggregation effectively promotes the collision of larger particles, because the collision velocity significantly depends not only on the velocity gradient, but also on the particle size.

When particles of different sizes and densities settle in the suspension, different settling rates can promote particle aggregation. Larger particles settle faster and are more likely to capture smaller particles when they settle.

Assuming that particles fall vertically before colliding with other particles, the simplest estimate of the collision frequency for spherical particles of the same density is based on Stokes' law: 


$$
k_{i, j}=\left(\frac{2 \pi g}{9 \eta}\right)\left(\rho_{s}-\rho\right)\left(a_{i}+a_{j}\right)^{3}\left(a_{i}-a_{j}\right),
$$

where $\rho$ - fluid density, $g$ - acceleration due to gravity, $a_{i}>a_{j}$.

Accordingly, differentiated sedimentation mainly depends on the size and density of the particles, and this transport mechanism is dominant, especially when the particles are large and dense. This process usually occurs at a later stage of flocculation, when aggregates of different sizes are formed, which settle at different rates.

An approach similar to molecular diffusion was used to determine the collision probability of two particles. Let's consider a dispersed medium containing in units of volume $N_{0}$ spherical particles of the same size (fig. 1 ). $N_{0}$ is such that the number of collisions of more than 2 particles is excluded. The liquid, by definition, is stationary.

One of the particles with $a$ diameter (test) will be considered stationary. If we define a sphere with radius $R$ around this particle such that $\left(\frac{a}{2}<R<<H\right)$, where $H$ - the distance between the centers of the nearest particles, we believe that every second particle crossing the surface of this sphere is captured by the investigated particle. Accordingly, on the surface $R$ the concentration of particles will be zero. On the surface $R$, as a result there is a concentration gradient of particles.

Then the Brownian flow of particles to $R$ will be described by an analogue of the molecular diffusion equation (diffusion equation):

$$
\frac{\partial n}{\partial t}=D \frac{1}{r} \frac{\partial}{\partial r}\left(r^{2} \frac{\partial n}{\partial t}\right)
$$

where $r$ - spherical coordinate with the center in the center of the investigated lobe; $D-$ the coefficient of "Brownian" diffusion of particles to the surface $R$.

In extreme conditions:

$$
n=n_{0} \Rightarrow r>R ; t=0 n=0 \Rightarrow r=R ; t>0 n=n_{0} \Rightarrow r \rightarrow \infty \text {. }
$$

Integrating the problem, we get an expression of the flow of particles through the surface, which determines the number of collisions of "rapid coagulation" with part of the test:

$$
J=D\left(\frac{\partial n}{\partial t}\right)_{r=R}=\frac{D n_{0}}{R}\left[1+\frac{R}{\sqrt{\pi D t}}\right] .
$$

For coagulation that does not occur in an organized stream of dispersed medium when the liquid is in a "quasi-equilibrium state", it will meet the condition:

$$
\underline{U}=\frac{G}{\rho F} \ll v_{\lambda}
$$

where $\underline{U}$ - macroscopic speed scale; $G$ - fluid flow; $\rho$-fluid density; $F$ - the cross-sectional area of the apparatus; $v_{\lambda}$ - internal scale of turbulent pulsations.

We will analyze this problem under the following conditions:

- the radius of coagulation of the investigated particle is much smaller than the internal

scale of turbulence $\lambda=\left(\frac{v^{3}}{\varepsilon}\right)^{\frac{1}{4}}, \varepsilon$-the rate of scattering of the kinetic energy of turbulence; $v$ - kinematic viscosity of the liquid;

- all particles are involved in the rapid exchange of energy;

- turbulence is isotropic, homogeneous;

- the problem has a stationary quasi-equilibrium character.

Because turbulent isotropic motion is disordered, the motion of particles is similar to and superimposed on Brownian motion. That is, particles coagulate simultaneously by Brownian and turbulent isotropic mechanisms.

By analogy with Brownian motion, we introduce, according to Bussinesque's hypothesis, the concept of "turbulent diffusion coefficient", and we formulate the diffusion problem under the condition of a stationary process. [27]: 


$$
\operatorname{div}\left(D_{e f f} \operatorname{gradn}\right)=0 .
$$

Since the problem is fixed, $n-$ is the average time of particle concentration time. Then the effective diffusion coefficient Deff according to previous statements will be following:

$$
D_{\text {eff }}=D+D_{\mathrm{T}} \text {, }
$$

where $\quad D-$ molecular diffusion coefficient, the so-called "Brownian"; $D_{\mathrm{T}}-$ turbulent diffusion coefficient.

Boundary conditions of the task:

$$
n=0 \Rightarrow r=R ; n=n_{0} \Rightarrow r \rightarrow \infty \text {. }
$$

To determine the diffusion coefficient $D_{\mathrm{T}}$ we use the analogy between energy pulse transmission and mass. Then, according to L. Prandtl's theory of "mixing path length" [28] $D_{\mathrm{T}}-$ can be written as follows:

$$
D_{\mathrm{T}} \approx v^{\prime} l,
$$

where $\quad v^{\prime}$-average pulsation of speed; $l$ - the length of the mixing path.

This expression is similar to the expression for molecular diffusion obtained in molecular kinetic theory, however, in contrast to the well-defined path length of the molecule $l_{\mathrm{T}}$ for turbulent motion, the length of the mixing path is not clearly defined. The pulsation velocity can be determined less clearly.

Based on the fact that only three-dimensional vortices with a characteristic size $l$ and a certain difference in velocities $\underline{\underline{U}}$, can overcome the inertial-gravitational forces in the liquid, and according to the scale 1 turbulence will be three-dimensional isotropic. According to Kolmogorov's theory of local-isotropic turbulence [29] $\Delta \underline{U}$ is written in the form :

$$
\Delta \underline{U} \sim(\varepsilon l)^{1 / 3} .
$$

It should be noted that in Kolmogorov's statistical theory the parameters of turbulence are determined on the basis of dimensional considerations and the hypothesis of stepwise energy transfer from the largest to the smallest vortex in which the kinetic energy of turbulence is scattered.

If the turbulence to a certain determinant scale $l_{o}$ is three-dimensional isotropic, then the only determinant parameters for processes not exceeding this scale will be $\varepsilon$ and $l$. In this case, for dimensional reasons, we obtain the isotropic diffusion coefficient:

where $K_{1}$ - some universal dimensionless constant.

$$
D_{\mathrm{T}}=K_{1} \varepsilon^{1 / 3 /} l^{4 / 3},
$$

This expression is obviously obtained for the rangel $>\lambda$, when reaching the size in the diffusion rangel $\approx \lambda$ small-scale vortices begin to play an active role in energy dissipation.

According to equation (14) the turbulence coefficient increases with increasing process scale to power $4 / 3$. It is obvious that this process will take place on the scale $l_{0}$, after which the growth of $D_{\mathrm{T}}$ should stop. Accordingly, the maximum value of $D_{\mathrm{T}}$ will look like:

$$
D_{\mathrm{T}}^{\max }=K_{1} \varepsilon^{1 / 3 /} l_{0}^{4 / 3} .
$$

However, in this range $l>\lambda$ the value $D_{\mathrm{T}}$ significantly exceeds the diffusion-molecular component $D$, respectively, the main diffusion resistance is in the region of small scales $l<$ $\lambda$ we can conclude that in the range $l>\lambda$ the gradient concentration of particles goes to zero from $l=\infty$.

In the range $\frac{a}{2}<l<\lambda$ the diffusion turbulence coefficient decreases in the range, and, according to Prandtl's theory of "mixing path length", we obtain the expression:

$$
0<D_{\mathrm{T}}<K_{1} \varepsilon^{1 / 3 /} \lambda^{4 / 3},
$$

According to Kolmogorov's theory, this range can be conditionally called the region of "turbulence attenuation", and Le Chatelier-Brown's "principles of mobile thermodynamic 


\section{- Processes, Equipment and Control Systems}

equilibrium" in the form of "attenuation equation" ("relaxation equation") can be used to analyze changes in turbulent transfer coefficient. [30].

Let's enter dimensionless variables:

$$
l^{\prime}=\frac{l}{a / 2} ; \quad D_{\mathrm{T}}^{\prime}=\frac{D_{\mathrm{T}}}{D_{\mathrm{T} \infty}} .
$$

The attenuation coefficient is defined as:

$$
z=1-D_{\mathrm{T}}^{\prime} \text {. }
$$

The attenuation equation and the boundary condition are written in the form, respectively:

$$
\frac{d z}{d l}=c_{1} z \text { and } z=1 \text { at } l^{\prime}=1 .
$$

As a result, the turbulent diffusion coefficient is written as:

To determine $c_{1}$ we write the condition:

$$
D_{\mathrm{T}}^{\prime}=1-z=1-\exp \left[c_{1}\left(l^{\prime}-1\right)\right] \text {. }
$$

$$
D_{\mathrm{T}}=D_{\mathrm{T} \lambda}=K \varepsilon^{1 / 3 /} \lambda^{4 / 3} \text { at } l^{\prime}=l_{\lambda}^{\prime}=\frac{\lambda}{a / 2} .
$$

As a result, we get:

$$
c_{1}=\frac{\ln \left(1-D_{\mathrm{T} \lambda}^{\prime}\right)}{l_{\lambda}^{\prime}-1} .
$$

As a result of approximation of the above equations by the "pseudo-Newtonian method" [30] within:

$$
\frac{a}{2}<l^{\prime}<\lambda, \text { where } \frac{a}{2} \approx 10^{-7} m ; \lambda \approx 10^{-5} m ; D_{\mathrm{T} \lambda} \approx 0,8 D_{\mathrm{T} \infty} .
$$

we obtain the equation of the coefficient of turbulent diffusion:

$$
D_{\mathrm{T}}^{\prime}=0,0368\left(l^{\prime}\right)^{0,677},
$$

Consider the particle concentration field in the region $\frac{a}{2}<l<\lambda$. In this region $D_{\mathrm{T}}$ decreases as expected with decreasing turbulent pulsations. It is obvious that at a certain value of the pulsation scale $D_{\mathrm{T}}$ becomes less than $D_{b r}$. If you define this scale as:

then at $l<l_{1} \Rightarrow D_{\mathrm{T}}<D_{b r}$;

$$
D_{\mathrm{T} \lambda} 0,0368\left(\frac{l_{1}}{\lambda}\right)^{0,677}=D_{b r}
$$

at $l_{1}<l \Rightarrow D_{b r}<D_{\mathrm{T}}$.

That is, if the coagulation radius around the particle $R$ is such that $R>l$, then preferably the diffusion is carried out by a turbulent mechanism.

Consider the case:

$$
R>l_{1}=\left(27,2 \cdot \frac{D_{b r}}{D_{\mathrm{T} \lambda}}\right)^{1,48} \cdot \lambda .
$$

Assuming that the solution of the boundary value problem is determined only by the radius of the vector $r$, we integrate the transfer equation into cylindrical coordinates under the appropriate boundary conditions::

$$
\frac{1}{r} \frac{\partial}{\partial r}\left(D_{e f} r \frac{\partial n}{\partial r}\right)=0
$$

The first integration allows you to get $R>l_{1}$ :

$$
D_{\mathrm{T} 1} r^{2} \frac{d n}{d r}=C \text {. }
$$

Substituting the value of $D_{\mathrm{T}}$ for the corresponding areas, we obtain:

$$
\begin{gathered}
K_{1} \varepsilon^{1 / 3 /} r^{10 / 3} \frac{d n}{d r}=C_{2} ; \text { at } r>\lambda ; \\
0,037 K_{1} \varepsilon^{0.17} v^{1,95} r^{2.68} \frac{d n}{d r}=C_{3} ; \text { at } r<\lambda .
\end{gathered}
$$

The integration of the last expression must be performed using numerical methods due to the complexity of the two-layer scheme with conjugation of flows at the layer boundary. 


\section{— Processes, Equipment and Control Systems -}

For a preliminary analysis, we simplify the calculation scheme by approximating the dependence of $r<\lambda$ on the domain by a linear function and assuming that $n=n_{0}$ at $r=$ $\lambda \zeta$.

Due to the qualitative nature of the analysis, these simplifications will give a rough picture of the process, which gives a general idea of the nature of the particle diffusion process.

As a result of integration for the range $R<r<\lambda$ we obtain the expression for the particle concentration field:

$$
n=\frac{n_{0} R^{2} \lambda^{2}}{\lambda^{2}-R^{2}}\left(\frac{1}{R^{2}}-\frac{1}{r^{2}}\right)
$$

Particle flux density on the surface of the coagulation sphere $r=R$ :

where $K_{1}=8 \cdot 10^{-3} K \varepsilon^{0,17} v^{1,95}$;

$$
j=D_{\mathrm{T}}\left(\frac{\partial n}{\partial r}\right)_{r=R}=K_{2} n_{0} \frac{1}{R} K_{1} R=K_{1} K_{2} n_{0},
$$

$$
K_{2}=\frac{R^{2} \lambda^{2}}{\lambda^{2}-R^{2}}
$$

That is, the particle flux density through the coagulation sphere depends only to a small extent on the size of the sphere.

At the same time, the total flow of particles, the intensity of "rapid" coagulation by the turbulent mechanism, will be defined as:

$$
N_{\mathrm{T}}=4 \pi R^{2} j=4 \pi R^{2} n_{0} K_{1} K_{2} .
$$

When the value of the dissipation rate of the kinetic energy of turbulence is of the order of $10^{2}-10^{4}\left(\frac{m}{s}\right)^{2}$ the value $R>l_{1}$ and, accordingly, $N_{\mathrm{T}}>N_{, r}$ for particles whose size in the mechanism is $10^{-7}-10^{-8} \mathrm{~m}$ coagulation of small particles is dominated by Brownian particle diffusion mechanism. That means, the more intense the process of mixing the suspension is, the greater the role in the process of coagulation, precipitation and filtration play particles of the nanoscale range, which are included in the rapid movement of the vortex.

\section{Conclusion}

This work provides a brief overview and analysis of mathematical models of aggregation kinetics in multiphase dispersed systems based on modifications of the particle dynamics method taking into account Van der Waals forces, gravity, Brownian and Stokes forces.

Based on the analysis of the Smolukhovsky equation, molecular kinetic and localisotropic turbulence of Kolmogorov's theories, the dynamics of simultaneous turbulent and Brownian transfer to the process of "fast coagulation" and the mechanism of influence of nanoparticles on their aggregate formation are investigated ability to colloidal heterogeneous dispersion.

The development of equations for the rate of kinetic energy scattering in a suspension makes it possible to determine the particle dispersion in which the Brownian diffusion of particles in the coagulation mechanism predominates. It means, the more intense the process of mixing the suspension is, the greater the role in the process of coagulation, precipitation and filtration play particles of the nanoscale range, which are included in the turbulent vortex motion. These results are useful for practical application in controlling the intensity of coagulation processes in mixing devices. 


\section{References}

1. Sergeev G.B. (2001), Nanohimija metallov, Uspehi himii, 70(10), pp. 915-933.

2. Ulberh Z., Hruzina T., Karpov O. (2008), Nanotekhnolohii v medytsyni: rol koloidnokhimichnykh protsesiv, Visnyk NAN Ukrainy, 8, pp. 28-41.

3. Christian P., Kammer V., Balousha P., Hofman Th. (2008), Nanoparticles: structure, properties, preparation and behavior in environmental media, Ecotoxicology, 17, pp. 326-343.

4. Hasselov M., Readmen J.W., Ranville J.F., Tiede K. (2008), Nanoparticles and characterization methodologies in environmental risk assessment of engineering nanoparticles, Ecotoxicology, 14, pp. 344-361.

5. Chen J.Ch., Wiley B.J., Xia Yu. (2007), One-dimensional nanostructures of metals: large-scale synthesis and some potential applications, Langmir, 23, pp. 4120-4129.

6. Manhong L., Xiaoping Y., Hanfan L., Weiyong Y. (2000), An investigation of the interaction between polyvinilpyrrolidone and metal cations, React. Funct. Polym., 44(1), pp. 55-64.

7. Tay F.E.H. (ed.) (2002), Microfluidics and BioMEMS Applications, Springer-Science, New York.

8. F.E.H. Tay (ed.), (2002), Microfluidics and BioMEMS Applications, Springer-Science, New York.

9. Liou W., Fang Y. (2005), Microfluid Mechanics: Principles and Modeling (Nanoscience and Technology), McGraw-Hill Education Publ., New York.

10. Karniadakis G.E., Beskok A., Aluru N. (2005), Microflows and nanoflows: Fundamentals and simulation, Springer-Science, New York.

11. Kleinstreuer C., Xu Z. (2016), Mathematical modeling and computer simulations of nanofluid flow with applications to cooling and lubrication. A review, Fluids, 1, pp. 16 48.

12. Kanagala H. K. (2013), Modeling of particle agglomeration in nanofluids. PhD Thesis, Lehigh University Press.

13. Srivastava G. S. (2012), Effect of aggregation on thermal conductivity and viscosity of nanofluid, Applied Nanosciences, 2, pp. 325-331.

14. Yu W., Xie H. (2012), A review on nanofluids: preparation, stability mechanisms, and applications, Journal of Nanomaterials, 2, pp. 435-473.

15. Lushnikov A. (1978), Evoljucija koagulirujushih sistem. D.Sc. in FMS, Moscow.

16. Netrebko N.I., Orlova I.V., Regirer S.A. (1987), Kvazistacionarnoe pul'sirujushhee techenie tiksotropnoj zhidkosti v cilindricheskoj trubke, Izv. AN USSR. Ser. MZhG, 1, pp. 3-9.

17. Pacegon N.F., Tarapov I.E., Fedonenko A.I. (1983), Issledovanie fizicheskih svojstv FMZh ul'trazvukovym metodom, Magnitn. Gidrodinamika, 4, pp. 53-59.

18. Pen'kov N.V. (1992), Koaguljacionnye processy v dispersnyh sistemah. D.Sc. in FMS, Moscow.

19. Phenrat T., Saleh N., Sirk K. (2007), Aggregation and sedimentation of aqueous nanoscale zerovalent iron dispersions, Eenviron. Sci. Technol., 41, pp. 284-290.

20. Lu J., Liu D., Yang X. (2015), Sedimentation of TiO2 nanoparticles in aqueous solutions: influence of $\mathrm{pH}$, ionic strength, and adsorption of hemic acid, Desalinate Water Treatment, 40(57), pp. 1-8.

21. Chung S.J., Leonard J.P., Nettleship I. (2009), Characterization of ZnO nanoparticle suspension in water: Effectiveness of ultrasonic dispersion, Powder Technol., 194, pp. 75-80. 
22. Jiang W., Ding G., Peng H., Hua H. (2010), Modeling of nanoparticles' aggregation and sedimentation in nanofluid, Current Appl. Phys., 10, pp. 934-941.

23. Ganguly S., Chakraborty S. (2011), Sedimentation of nanoparticles in nanoscale colloidal suspensions, Phys. Lett., Ser. A, 375, pp. 2394-2399.

24. Markus A.A., Parsons J.R., Roex E. W. M. (2015), Modeling aggregation and sedimentation of nanoparticles in the aquatic environment, Sci. Total Envir., pp. 506507, pp. 323-329.

25. Smoluchowski M. (2017), Mathematical theory of the kinetics of the coagulation of colloidal solutions, Z. Phys. Chem., 92, 129-168.

26. Hari Krishna K., Neti S., Oztekin A., Mohapatra A. (2015), Modeling of particle agglomeration in nanofluids, Journal of Applied Physics, pp. 117-125.

27. Sharfarec B. P., Dmitriev S. P. (2018), Modelirovanie turbulentnogo dvizhenija zhidkosti na osnove gipotezy Bussineska. Nauchnoe Priborostroenie, 3(28), pp. 101108.

28. Lezhenkin O. M., Samoichuk K.O, Kovalov O.O., Palianychka N.O., Verkholantseva V.O. (2017), Vyznachennia shliakhu zmishuvannia ta dotychnykh napruzhen v strumynnomu homohenizatori moloka, Visnyk Ukrainskoho viddilennia Mizhnarodnoi akademii ahrarnoi osvity, 5, pp. 129-142.

29. Kovalchuk M., Hirniak M., Laba I. (2008), Vyznachennia parametriv dribnomasshtabnoi turbulentnosti v aktyvnykh oblastiakh na sontsi dlia prohnozu spalakhiv, Visnyk Lviv. Un-tu. Seriia fizychna, 41, pp. 61-67.

30. Tkachenko V. G. (2016), Fizicheskie osnovy dlitel'noj prochnosti tvjordyh rastvorov s razlichnoj kinetikoj podvizhnyh defektov, Uspehi fiz. Met, 17, pp. 173-200.

31. Kalion V. A. (2016), Obchysliuvalna hidromekhanika. Rivniannia Navie-Stoksa, Kyiv. 